\title{
Specific induction of neuronal cells from bone marrow stromal cells and application for autologous transplantation
}

\author{
Mari Dezawa, ${ }^{1}$ Hiroshi Kanno, 2 Mikio Hoshino, 3,4 Hirotomi Cho,,5 Naoya Matsumoto,1 \\ Yutaka Itokazu, ${ }^{1}$ Nobuyoshi Tajima, ${ }^{6}$ Hitoshi Yamada, ${ }^{7}$ Hajime Sawada, ${ }^{8}$ Hiroto Ishikawa, ${ }^{1}$ \\ Toshirou Mimura, ${ }^{2}$ Masaaki Kitada, ${ }^{1}$ Yoshihisa Suzuki, ${ }^{5}$ and Chizuka Ide ${ }^{1}$ \\ 1Department of Anatomy and Neurobiology, Kyoto University Graduate School of Medicine, Kyoto, Japan. ${ }^{2}$ Department of Neurosurgery, \\ Yokohama City University School of Medicine, Yokohama, Japan. ${ }^{3}$ Department of Pathology and Tumor Biology, \\ Kyoto University Graduate School of Medicine, Kyoto, Japan. 4PRESTO, Japan Science and Technology Agency, Kawaguchi, Japan. \\ ${ }^{5}$ Department of Plastic and Reconstructive Surgery, Kyoto University Graduate School of Medicine, Kyoto, Japan. ${ }^{6}$ Department of Bacteriology, \\ 7Department of Neurology, and ${ }^{8}$ Department of Anatomy, Yokohama City University School of Medicine, Yokohama, Japan.
}

\begin{abstract}
Bone marrow stromal cells (MSCs) have the capability under specific conditions of differentiating into various cell types such as osteocytes, chondrocytes, and adipocytes. Here we demonstrate a highly efficient and specific induction of cells with neuronal characteristics, without glial differentiation, from both rat and human MSCs using gene transfection with Notch intracellular domain (NICD) and subsequent treatment with bFGF, forskolin, and ciliary neurotrophic factor. MSCs expressed markers related to neural stem cells after transfection with NICD, and subsequent trophic factor administration induced neuronal cells. Some of them showed voltage-gated fast sodium and delayed rectifier potassium currents and action potentials compatible with characteristics of functional neurons. Further treatment of the induced neuronal cells with glial cell line-derived neurotrophic factor (GDNF) increased the proportion of tyrosine hydroxylase-positive and dopamine-producing cells. Transplantation of these GDNF-treated cells showed improvement in apomorphine-induced rotational behavior and adjusting step and paw-reaching tests following intrastriatal implantation in a 6-hydroxy dopamine rat model of Parkinson disease. This study shows that a population of neuronal cells can be specifically generated from MSCs and that induced cells may allow for a neuroreconstructive approach.
\end{abstract}

\section{Introduction}

Many of the neurodegenerative disorders are attributed to degeneration of specific neurons with subsequent functional loss. Cell replacement is a potential strategy for the treatment of such diseases, and ES cells, neural stem cells (NSCs), and neuronal progenitor cells (NPCs) have been proposed as candidates for transplantation therapy in human neural diseases (1). They may face limitations inherent in procurement from fetal tissue, however, including problems of histocompatibility, inadequate tissue supply and therapeutic scale, and ethical concerns. Furthermore, there is the problem of contamination by glial cells during neural induction.

Bone marrow stromal cells (MSCs) have the capability under specific conditions to differentiate into other cell types such as osteoblasts, adipocytes, and chondrocytes (2). They also can

Nonstandard abbreviations used: anteroposterior (A/P); artificial cerebrospinal fluid (ACSF); bone marrow stromal cells (MSCs); brain-derived neurotrophic factor (BDNF); ciliary neurotrophic factor (CNTF); dopamine transporter (DAT); forskolin (FSK); galactocerebroside (GalC); glial cell line-derived neurotrophic factor (GDNF); glial fibrillary acidic protein (GFAP); hairy/enhancer of split-1 (Hes1); 6-hydroxy dopamine (6-OHDA); Janus kinase (JAK); lateral (L); microtubule-associated protein-2ab (MAP-2ab); nerve growth factor (NGF); neural stem cell (NSC); neuronal progenitor cell (NPC); NICD-transfected MSC (N-MSC); N-MSC administrated with the trophic factors (TF-MSC); Notch intracellular domain (NICD); 3-phosphoglycerate dehydrogenase (3-PGDH); signal transducer and activator of transcription 1 (STAT1); tetrodotoxin (TTX); TF-MSC administrated with GDNF (G-MSC); tyrosine hydroxylase (TH); ventral (V).

Conflict of interest: The authors have declared that no conflict of interest exists.

Citation for this article: J. Clin. Invest. 113:1701-1710 (2004).

doi:10.1172/JCI200420935 differentiate into neural lineages. Several reports in vitro have shown neuronal cell induction from MSCs with some accompanying induction of glial cells $(3,4)$ and have also shown that MSCs could be induced to form dopamine decarboxylase-positive cells by exposing them to FGF-2, FGF-8, and brain-derived neurotrophic factor (BDNF) (5). The induction ratio of neurons is not satisfactorily, however, and the underlying molecular mechanism of neuronal differentiation of MSCs has not yet been clarified. Furthermore, functional recovery with transplantation of these induced neural cells in animal models of neuronal diseases has not been tested.

The Notch gene encodes a 300-kDa single transmembrane cell surface receptor protein that is activated by Delta/Serrate/Lag-1 ligands presented by neighboring cells (6). Upon ligand binding, the intracellular portion of the Notch receptor is cleaved and enters the nucleus, where it influences the expression of numerous transcription factors related to progenitor pool maintenance, cell fate decision, and, in case of nervous system, terminal specification of cells as neurons and glial cells (6-8).

In this experiment, a highly efficient, exclusive, and specific induction of postmitotic neuronal cells was achieved in MSCs by gene transfer of Notch intracellular domain (NICD) followed by the administration of certain trophic factors. Additional administration of glial cell line-derived neurotrophic factor (GDNF) to those cells can efficiently induce tyrosine hydroxylase-positive (TH-positive) and dopamine-producing cells. Furthermore, functional recovery could be achieved when grafted in a rat model of Parkinson disease, suggesting that 

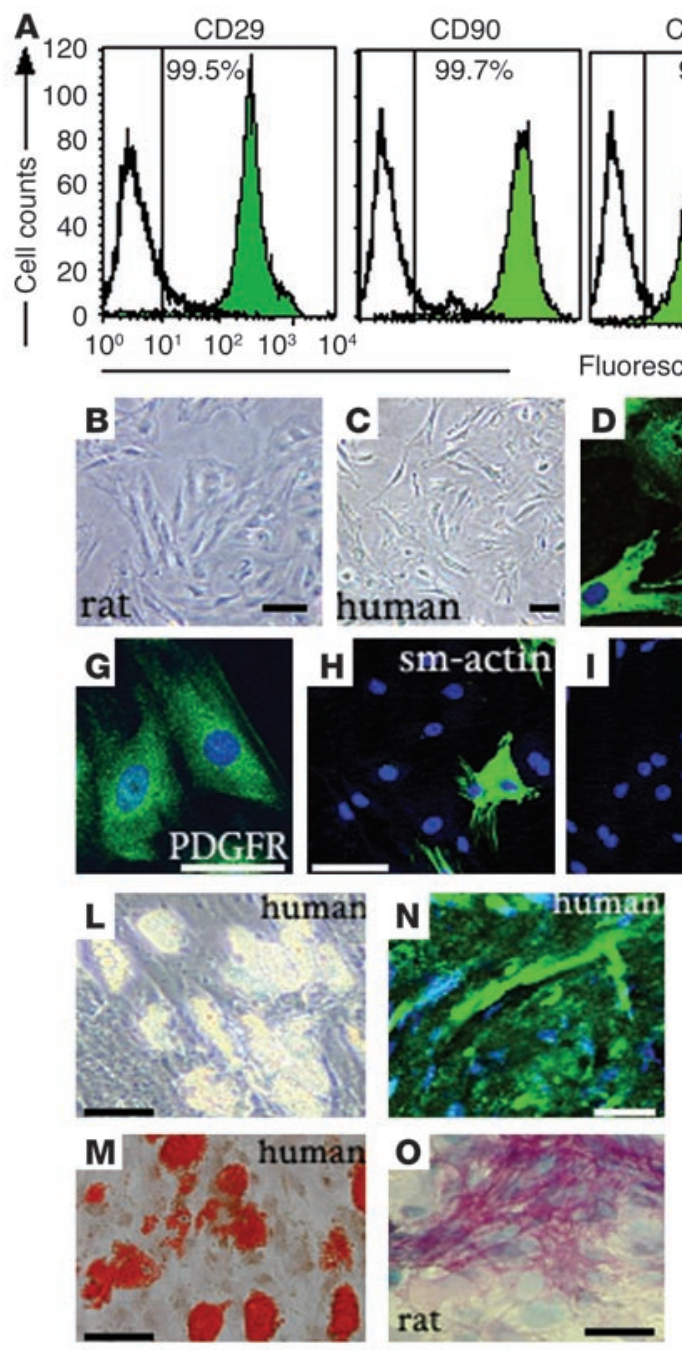

Fluorescence intensity
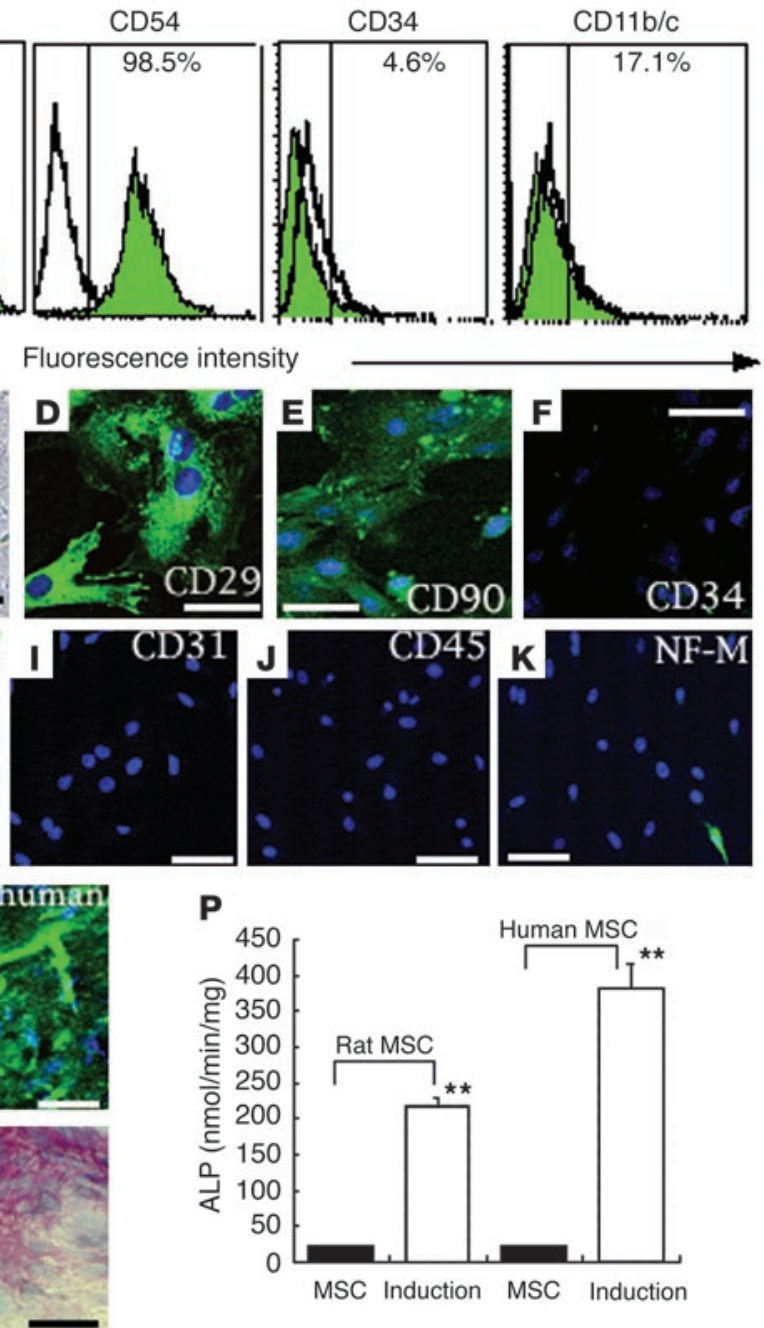

Figure 1

Characterization of isolated MSCs. (A) FACS analysis of rat MSCs. Numbers in panels represent mean fluorescent intensity of the cells expressing each marker. (B and $\mathbf{C}$ ) Phase-contrast microscopy of rat (B) and human (C) MSCs. (D-K) Immunocytochemistry of CD29 (D), CD90 (E), and CD34 (F) of human MSCs and PDGF receptor $\beta$ (PDGFR) (G), and smooth muscle actin (sm-actin) (H), CD31 (I), CD45 $(\mathbf{J})$, and neurofilamen-M (K) of rat MSCs. Bars $=50 \mu \mathrm{m}$. (L-P) Adipogenic ( $\mathbf{L}$ and $\mathbf{M})$, chondrogenic $(\mathbf{N})$, and osteogenic $(\mathbf{O})$ induction from human (L-N) and rat (0) MSCs. Micrographs in (M) show oil red staining of lipid droplet in adipocytes in (N) immunocytochemistry of collagen type II of chondrogenic induction and in (0) alkaline phosphatase in osteocytes. (L) Phase-contrast image of adipocytes. Bars $=50 \mu \mathrm{m}$. (P) Alkaline phosphatase (ALP) activity of rat and human MSCs before and after osteogenic induction. ${ }^{\star \star} P<0.01$. these induced cells may have properties useful in the treatment of neurodegenerative disorders such as Parkinson disease.

\section{Results}

Characterization of isolated MSCs. Cell surface markers were assessed using FACS to characterize isolated rat and human MSCs. MSCs expressed CD29 ( $\beta 1$-integrin), CD90 (Thy-1), and CD54 (ICAM-1), but not CD34 (a hematopoietic stem cell marker), CD11b/c (macrophages), or vWF (human endothelial cells; not shown), consistent with previous reports $(2,9)$ (Figure 1A). Basically identical results were obtained by immunocytochemical examination (Figure 1, B-F). MSCs were also immunopositive to mesenchymal progenitor markers; $95.3 \% \pm 6.5 \%$ showed positivity to PDGF receptor $\beta$ and $43.7 \% \pm 4.0 \%$ to smooth muscle actin (Figure $1, \mathrm{G}$ and $\mathrm{H})$. In contrast, vascular (CD31) and hematopoietic (CD45, leukocyte common antigen) lineage markers were negative (Figure 1, I and J). As for neuronal markers, MSCs did not show positivity to microtubule-associated protein-2ab (MAP-2ab) and $\beta$-tubulin isotype 3 , while $0.3 \% \pm 0.1 \%$ of cells were immunoreactive to neurofilament-M (Figure 1K). Adipogenic (Figure 1, L and M), chondrogenic (Figure 1N), and osteogenic (Figure 1, O and P) differentiations of both rat and human MSCs were confirmed according to the method described by Pittenger et al. (2), further indicating that these cells were of mesenchymal origin. While MSC populations showed no oil red-positive adipocytes, with induction their proportion among human MSCs could become as high as $25.5 \% \pm 1.3 \%$ (Figure 1M). Chondrogenesis of human MSCs could be shown by staining with an $\mathrm{Ab}$ to collagen type II (Figure $1 \mathrm{~N}$ ). Finally, osteogenic induction of rat and human MSCs was shown by a substantial increase of alkaline phosphatase activity (Figure 1, $\mathrm{O}$ and $\mathrm{P})$. These results confirmed that the isolated cells exhibited previously reported properties of MSCs $(2,9)$.

NICD-transfected MSCs. We transfected MSCs with NICD by lipofection followed by G418 selection. We refer to the NICDtransfected MSCs as N-MSCs here. The efficiency of NICD transfection was assessed by lipofection of pNICD-IRES2-EGFP, a GFP gene containing plasmid, showing that $98.8 \% \pm 0.8 \%$ of cells were transfected with NICD after G418 selection.

We examined the expression of glutamate transporter GLAST, 3phosphoglycerate dehydrogenase (3-PGDH), and nestin, which have been found to be expressed in NSCs and NPCs and in radial glia $(10,11)$. Rat MSCs showed little expression of GLAST and 3-PGDH, whereas N-MSCs substantially upregulated the expression of both of these molecules, with most N-MSCs becoming positive for GLAST and 3-PGDH (Figure 2A). We found that a very small fraction of MSCs were positive for nestin $(0.74 \% \pm 0.1 \%)$, while this increased to 
A
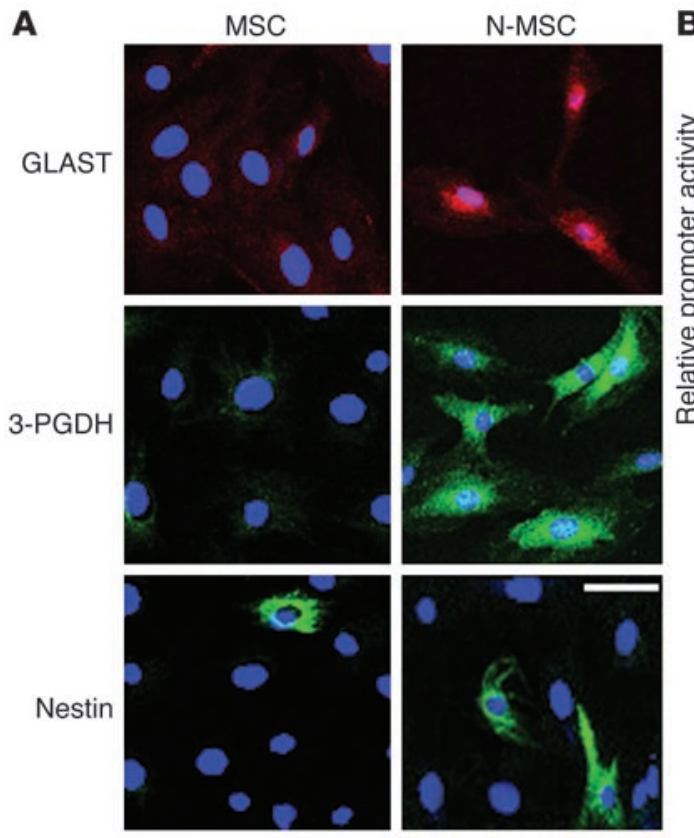

B

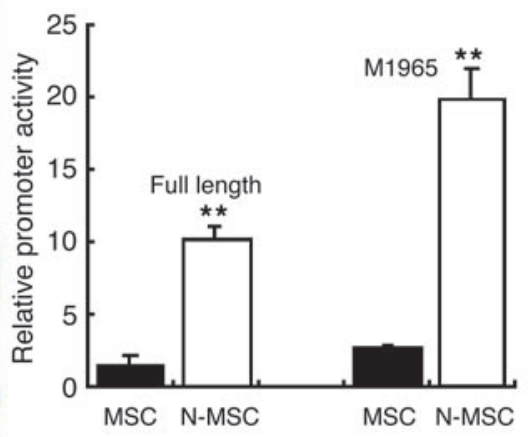

\section{Figure 2}

Phenotype of N-MSCs. (A) Immunocytochemistry of GLAST, 3-PGDH, and nestin in rat MSCs and N-MSCs. Bar $=50$ $\mu \mathrm{m}$. (B) Promoter activity of 3-PGDH in rat MSCs and N-MSCs (NICD transfection followed by 11 days of $\mathrm{G} 418$ selection). Both full-length and truncated (M1965) forms of 3-PGDH showed nine- to tenfold increases in promoter activity in N-MSCs. ${ }^{* *} P<0.01$.
$4.92 \% \pm 1.0 \%(P<0.01)$ in N-MSCs (Figure 2A). Luciferase promoter assay indicated that $5^{\prime}$-flanking/full-length (nucleotides -3472 to -1) and 5'-flanking/M1965 (-1792 to -1) 3-PGDH promoter activities, both reported to be active in radial glia and neuroepithelial cells (10), increased significantly in N-MSCs as well $(P<0.01)$ (Figure 2B). We found few glial fibrillary acidic protein-positive (GFAP-positive) cells in the N-MSC population (data not shown).

Trophic factor administration to N-MSCs. We examined what conditions were required to selectively generate neuronal cells from N-MSCs. For this purpose, several trophic factors (neurotrophins, leukemia inhibitory factor, bFGF, and ciliary neurotrophic factor [CNTF]) and forskolin (FSK) (known to upregulate intracellular cAMP) were tested.

N-MSCs were subcultured once (60-70\% confluence) with certain trophic factors for 5 days, followed by immunostaining with an MAP-2ab Ab (known to be a marker for postmitotic neurons) (Table 1). Certain combinations of trophic factors drove N-MSCs to be MAP-2ab positive; $96.5 \% \pm 0.6 \%$ with three trophic factors (FSK plus bFGF plus CNTF), $73.2 \% \pm 5.1 \%$ with bFGF alone, and $87.5 \% \pm 3.1 \%$ and $83.6 \% \pm 3.4 \%$ with FSK or CNTF, respectively. On the other hand, FSK alone and CNTF alone achieved $29.2 \% \pm 5.4 \%$ and $4.3 \% \pm 1.9 \%$, respectively $(P<0.01)$ and $11.4 \% \pm 2.4 \%$ together. Because the administration of the three trophic factors (FSK plus bFGF plus CNTF) marked the highest induction ratio of MAP2ab-positive cells, we refer to this induction condition as "trophic factor induction" and refer to the N-MSCs administrated with the three trophic factors as "TF-MSCs".

The induction of MAP-2ab cells by trophic factors did not seem to be caused by specific killing of non-neuronal cells, since few dead cells were observed by TOTO-3 nuclear staining following trophic factor induction (data not shown). Nontransfected as well as control vector-transfected MSCs could not be induced to be MAP2-ab positive by the trophic factor induction (Table 1).

Characterization of TF-MSCs. Rat and human TF-MSCs (5 days after trophic factor induction) showed neuritelike processes with abundant varicosities and expressed neuronal markers such as
MAP-2ab, neurofilament-M, and $\beta$-tubulin isotype 3 (Figure 3, A-F), although a few nestin-positive cells could also be recognized $(2.03 \% \pm 0.7 \%)$ (data not shown). Western blot analysis also confirmed that MAP-2ab was not present in MSCs but was found in TF-MSCs (Figure 3K). Triple staining of TF-MSCs with antiMAP-2ab, neurofilament-M, and $\beta$-tubulin isotype $3 \mathrm{Ab}$ 's showed that $89.4 \% \pm 4.5 \%$ of MAP- 2 ab cells were also expressing both neurofilament-M and $\beta$-tubulin isotype 3 (data not shown). Few cells were immunopositive to CD31 and CD 45 , while $3.4 \% \pm 1.1 \%$ and $2.8 \% \pm 0.7 \%$ were positive for PDGF receptor $\beta$ and smooth muscle actin, respectively (data not shown).

These TF-MSCs did not proliferate when subcultured after trypsin treatment. Brd-U incorporation studied 5 days after trophic factor induction showed few BrdU-labeled MAP-2ab-positive cells (Figure $3 \mathrm{G}$ ). In addition, approximately $0.14 \%$ of MAP-2abpositive cells were immunoreactive to intrinsic proliferation-asso-

\section{Table 1}

The ratio of MAP-2ab+ cells in each treatment

$\begin{array}{lcccc}\text { NICD transfection } & \text { FSK } & \text { bFGF } & \text { CNTF } & \% \text { of MAP-2ab+cells } \\ + & + & + & + & 96.5 \pm 0.6 \\ & & & & (\text { TF-MSCS) } \\ + & + & + & - & 87.5 \pm 3.1 \\ + & - & + & + & 83.6 \pm 3.4 \\ + & - & + & - & 73.2 \pm 5.1^{\mathrm{A}} \\ + & + & - & - & 29.2 \pm 5.4^{\mathrm{A}} \\ + & + & - & + & 11.4 \pm 2.4^{\mathrm{A}} \\ + & - & - & + & 4.3 \pm 1.9^{\mathrm{A}} \\ - & + & + & + & 0^{\mathrm{A}} \\ \text { pCl-neo control } & \text { FSK } & \text { bFGF } & \text { CNTF } & \% \text { of MAP-2ab+ } \\ \text { vector transfection } & & & & \text { cells } \\ + & + & + & + & 0^{\mathrm{A}}\end{array}$

AHighly significant difference $(P<0.01)$ to triple trophic factor induction (FSK + bFGF + CNTF). 


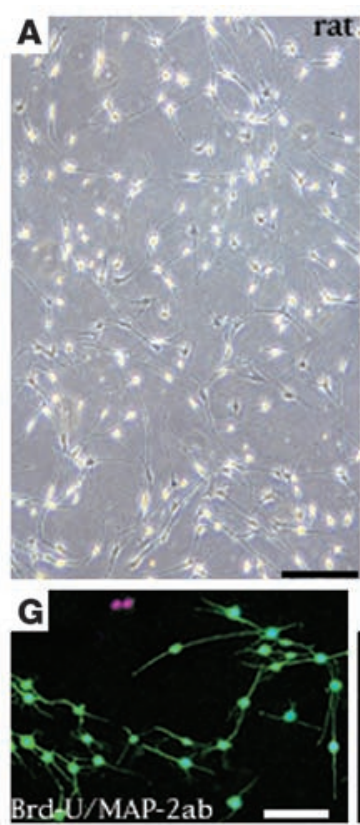

0

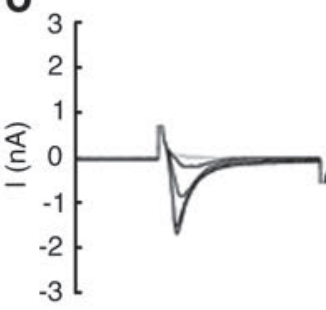

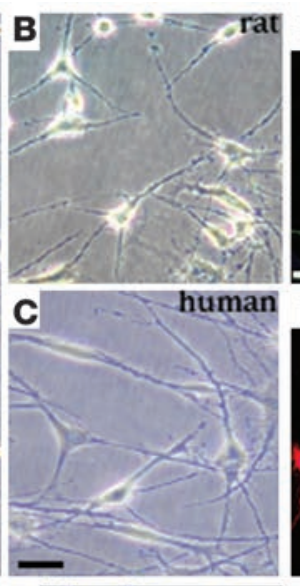
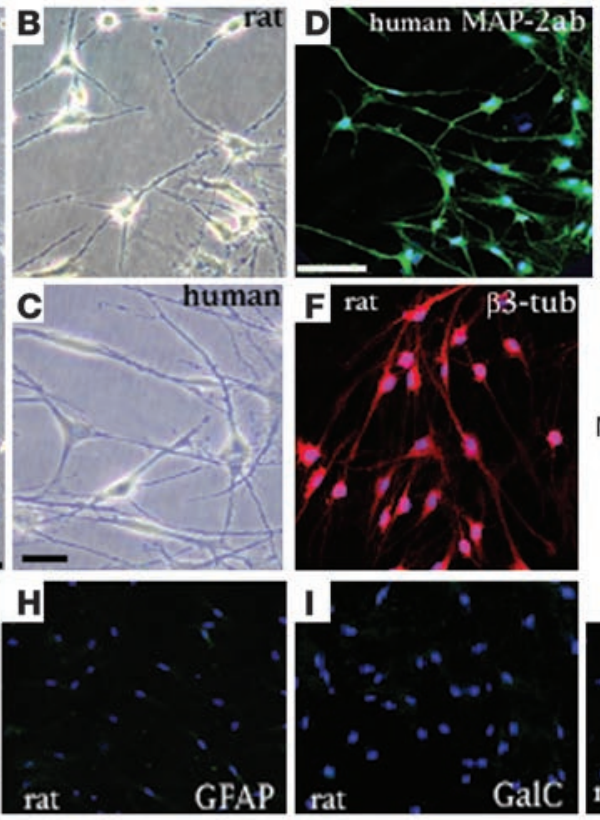

$\mathbf{P}$

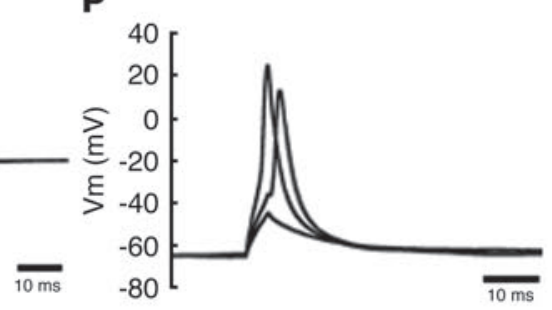

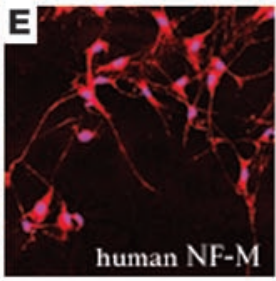
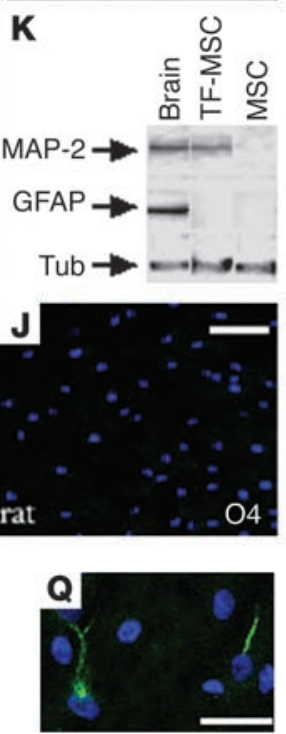

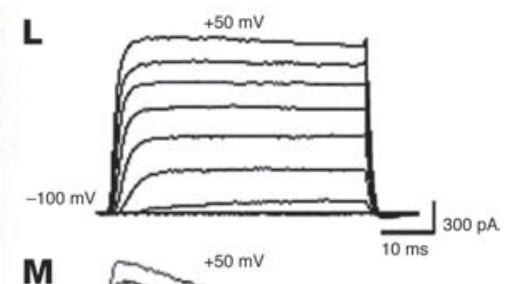

M
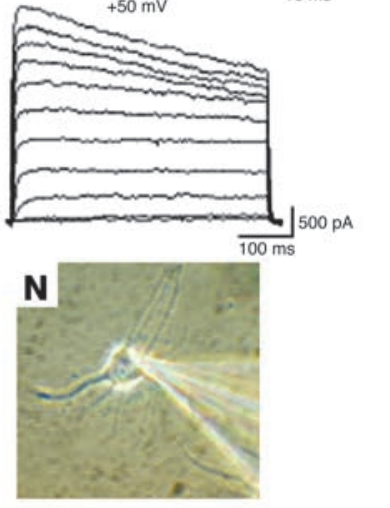

$\mathbf{R}$

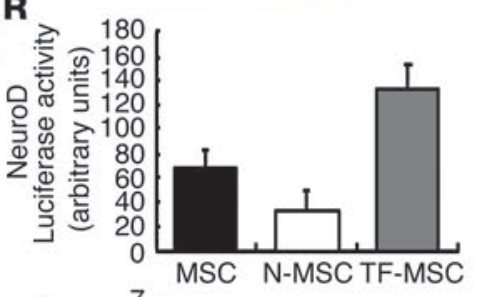

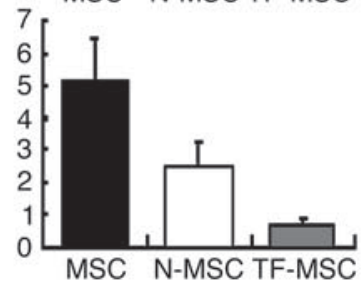

\section{Figure 3}

Analysis of TF-MSCs (5 days after trophic factor induction). (A-C) Phase contrast of TF-MSCs from rats $(\mathbf{A}$ and $\mathbf{B})$ and humans $(\mathbf{C})$. Bars, $\mathbf{A}=200$ $\mu \mathrm{m}, \mathbf{B}$ and $\mathbf{C}=50 \mu \mathrm{m}$. (D-F and $\mathbf{H}-\mathbf{J})$ Immunocytochemical analysis of neuronal and glial markers in rat $(\mathbf{F}, \mathbf{H}-\mathbf{J})$ and human (D and E) TF-MSCs. MAP-2ab (D), neurofilament-M (E), and $\beta$-tubulin isotype $3(\mathbf{F})$ were detected. None of the cells were reactive to GFAP (H), GalC (I), and O4 (J). (G) The Brd-U labeling of rat TF-MSCs. MAP-2ab-positive cells (green) did not incorporate Brd-U (red), whereas negative cells were occasionally incorporated with Brd-U. Bars in D-J = $100 \mu \mathrm{m}$. (K) Western blot analysis of MAP-2ab and GFAP rat samples. Brain, positive control; TF-MSC. $\beta$-tubulin (tub) as a loading control. (L-Q) Patch clamp. $\mathrm{K}^{+}$current increased with trophic factor induction up to approximately 1,600 pA and 4,000 pA in rat (L) and human (M) TF-MSCs, respectively. (N) Phase contrast of human TF-MSCs recorded in (M). (O) Voltage-gated inward current recorded in rat BDNF + NGF-treated TF-MSCs. A series of Na current to show the process of block by TTX. Capacity current was blanked. (P) Action potentials from rat BDNF + NGF-treated TF-MSCs; subthreshold, threshold, and suprathreshold current injections were made. (Q) Immunocytochemistry of sodium channel (green). Bar $=30 \mu \mathrm{m}$. (R) Relative promoter activities of NeuroD and GFAP in rat MSCs, N-MSCs, and TF-MSCs.

ciated marker Ki67 (data not shown). These results may suggest that large majority of TF-MSCs were mitotically terminated.

A rise in delayed rectifier potassium current is associated with the maturation of cell excitability and neuronal differentiation (12). We investigated this property in TF-MSCs by using the voltage-clamp method. An outwardly rectified $\mathrm{K}^{+}$current was elicited by positive voltage steps in TF-MSCs (7 days after trophic factor induction). In 51 out of 78 (65\%) rat TF-MSCs (Figure $3 \mathrm{~L}$ ) and in 32 out of 55 (58\%) human TF-MSCs (Figure 3, M and N), we detected the $\mathrm{K}^{+}$current amplitude, which was dramatically higher than that in control MSCs (data not shown). We also investigated resting membrane potential under current clamp conditions immediately after whole-cell configuration was formed. Resting membrane potentials were lower among TF-MSCs than in MSCs ( -50 to $-60 \mathrm{mV}$ and -30 to $-40 \mathrm{mV}$, respectively).

To evaluate expression of functional voltage-gated ion channels, we assessed the voltage-gated fast sodium currents by whole cell patch-recording in rat TF-MSCs. Inward currents could not be observed up to 14 days after trophic factor induction, and positive signals to the sodium channel could not be recognized in immunocytochemistry (data not shown). Because TF-MSCs exhibited neuronlike morphology and expressed several neuronal markers such as MAP-2ab, neurofilament-M, and $\beta$-tubulin isotype 3 , cells were speculated to be not fully maturated neurons 


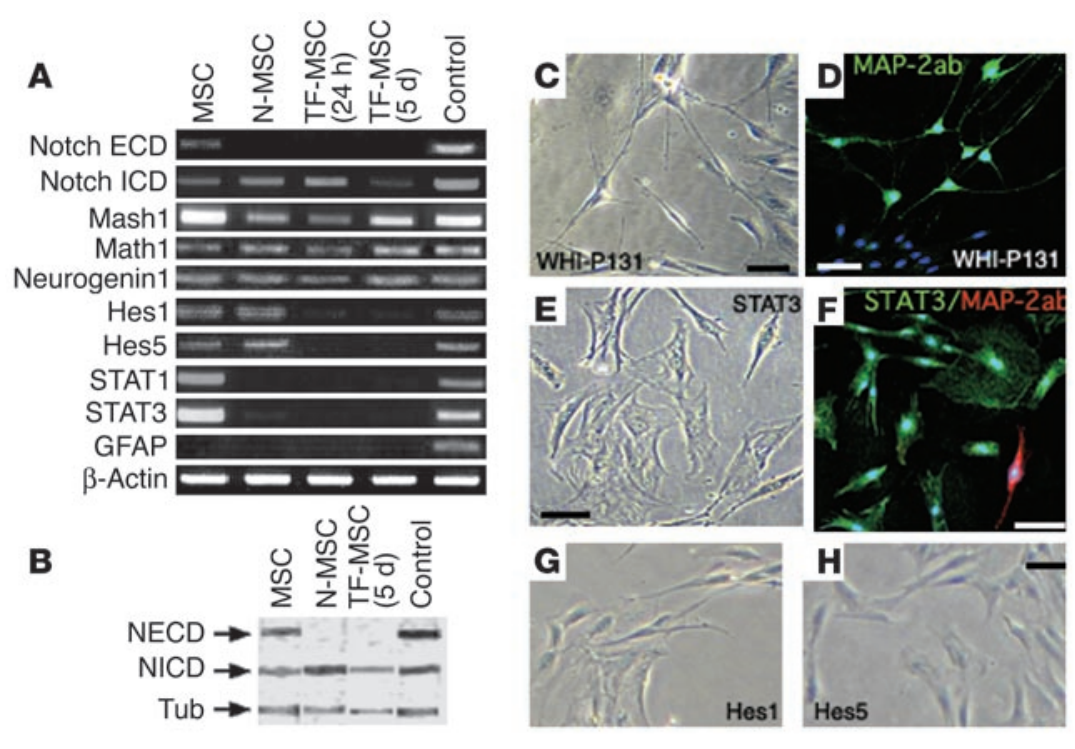

\section{Figure 4}

Transcription factors during induction. (A) RT-PCR of rat MSCs, N-MSCs, 24 hours and 5 days after trophic factor induction in TF-MSCs, and positive control. (B) Western blot of Notch extracellular domain (NECD) and NICD in rat MSCs, N-MSCs, TF-MSCs ( 5 days after trophic factor induction), and positive control from brain. $\beta$-tubulin (tub) as a loading control. (C and $\mathbf{D})$ Generation of MAP-2ab+ cells (green in D) by JAK/STAT inhibitor WHI-P131 administration and subsequent trophic factor induction in rat MSCs. ( $E$ and $\mathbf{F}$ ) Rat MSCs cotransfected with active form of STAT3 (anti-FLAG; green) and NICD, followed by trophic factor induction. Only a small number of cells were positive for MAP-2ab (red). ( $\mathbf{G}$ and $\mathbf{H}$ ) Hes $1 / 5$ transfection to rat MSCs. Bars in $\mathbf{C}-\mathbf{H}=80 \mu \mathrm{m}$. but rather to be in a process of maturation. Therefore, trophic factors of BDNF and nerve growth factor (NGF), both of which are known to promote neuronal maturation (13), were further administered to the culture media 7 days after trophic factor induction. Seven days after the addition of BDNF and NGF (14 days from trophic factor induction), tetrodotoxin-sensitive (TTX-sensitive) inward currents that may correspond to the sodium currents were detected in 16 out of 35 cells (Figure 3O). Furthermore, generation of the action potential was recorded in the BDNF- plus NGF-treated TF-MSCs 18 days after trophic factor induction (11 days after the administration of BDNF and NGF) (12 out of 30 cells; Figure 3P), and these cells were stained with anti-sodium channel, which resulted in $14.3 \%$ of positivity (Figure 3Q). These results may suggest that MAP-2ab-positive cells after the simple trophic factor induction, namely TF-MSCs, are neuronal cells in a premature state, which can be induced to be functionally mature neurons with further administration of certain trophic factors such as BDNF and NGF.

In checking for glial cells, we performed immunocytochemistry using GFAP as a marker for astrocytes and galactocerebroside (GalC) and $\mathrm{O} 4$ as markers for oligodendrocytes. No marker-positive glial cells were detected 5 days after trophic factor induction of rat or human TF-MSCs (Figure 3, H-J). This was confirmed by Western blot analysis (Figure $3 \mathrm{~K}$ ). To further confirm specificity of TF-MSCs, we measured the promoter activities of NeuroD and GFAP. In rat MSCs, relative promoter activities of NeuroD and GFAP were $67.2 \pm 15.3$ and $5.16 \pm 1.36$ Luciferase activity (arbitrary units), respectively. In N-MSCs, this activity decreased to $31.98 \pm 17.5$ and $2.49 \pm 0.15$. In TF-MSCs, however, NeuroD activity increased significantly to $132.7 \pm 20.9$, while GFAP decreased to $0.63 \pm 0.22$ (Figure 3R).

All above observations were carried out using MSCs transfected en masse. To examine if clonally expanded MSCs are also able to differentiate into neuronal cells, a single rat MSC was separated, expanded, transfected with NICD (pCI-neo vector), and then processed for trophic factor induction. Cell surface markers were analyzed by FACS, showing that they were positive for CD29, CD90, and CD54, but negative for CD34 and CD11b/c (data not shown). The final population was immunopositive to MAP- $2 \mathrm{ab}(95.6 \% \pm 0.3 \%)$,
neurofilament-M, and $\beta$-tubulin isotype 3 . There were no cells positive for GFAP, O4 and GalC. Only a small fraction $(2.1 \% \pm 0.8 \%)$ was nestin positive (data not shown).

Analysis of the induction event. To further examine the induction event from MSCs to TF-MSCs, we analyzed the expression of genes related to neural development using RT-PCR (14-18) (Figure 4A). In rat MSCs, neuronal basic helix-loop-helix Mash1, Math1, Neurogenin1, together with hairy/enhancer of split 1 (Hes1), Hes5, signal transducer, and activator of transcription 1 (STAT1) and STAT3 were detected. In N-MSCs, STAT1 and STAT3 expressions were downregulated, and subsequent trophic factor induction for 24 hours also downregulated the expression of Hes1 and Hes5. The expression of Mash1, Math1, and Neurogenin 1 could persistently be seen until 5 days after trophic factor induction. Increased expression of GFAP mRNA was not detected at any point (Figure 4A). Notch extracellular and intracellular domains were detected in MSCs, suggesting that MSCs are endogenously expressing a small amount of Notch (Figure 4A). As to endogenous Notch expression, extracellular domain was downregulated in N-MSCs and remained negative up to 5 days after trophic factor induction, indicating that the endogenous expression of Notch was downregulated by NICD introduction. NICD was detected throughout this period, although the expression declined slightly by 5 days after trophic factor induction (Figure 4A). Western blot confirmed the RT-PCR data of NICD and Notch extracellular and domains (Figure 4B).

The suppression of STATs after the introduction of NICD in N-MSCs may also be related to the induction of a neuronal phenotype. Therefore, the Janus kinase (JAK)/STAT inhibitor WHIP131 was administrated to MSCs for 2 days instead of NICD transfection, followed by trophic factor induction. Three days after trophic factor induction, $29.4 \% \pm 3.0 \%$ of MAP-2ab-positive cells with neuritelike processes were recognized (Figure 4, C and D). To see whether the ratio of MAP-2ab-positive cell is reduced or eliminated when an active form of STAT is introduced into MSCs, we cotransfected pEF-BOS FLAG-mouse STAT3 with NICD into rat MSCs, followed by trophic factor induction. STAT3 expression was confirmed by RT-PCR and immunocytochemistry of anti-FLAG $\mathrm{Ab}$ both after transfection and trophic factor induction (data not 


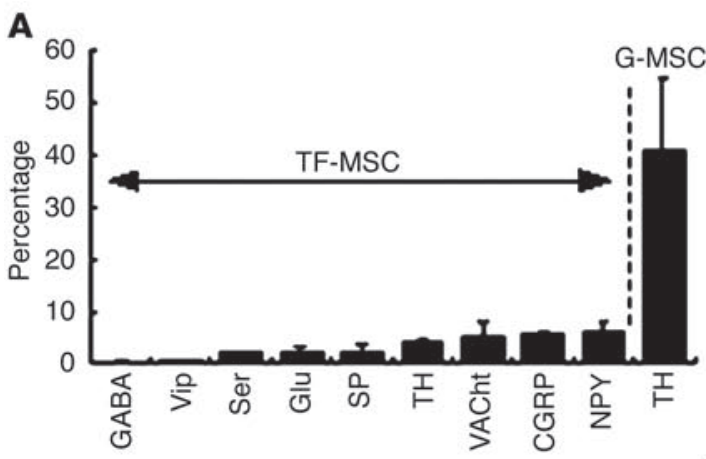

$\mathbf{E}$

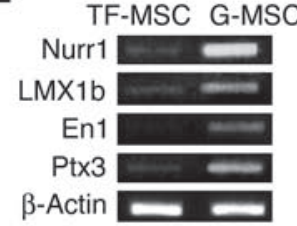

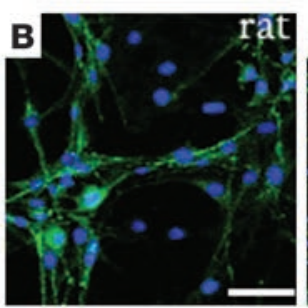
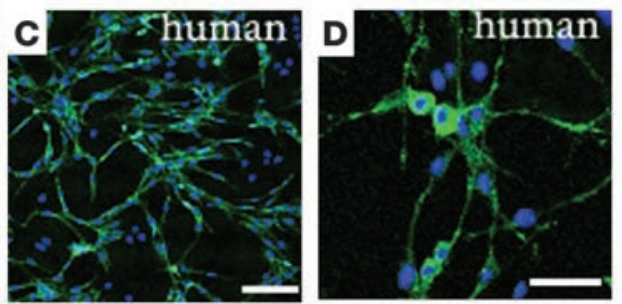

G

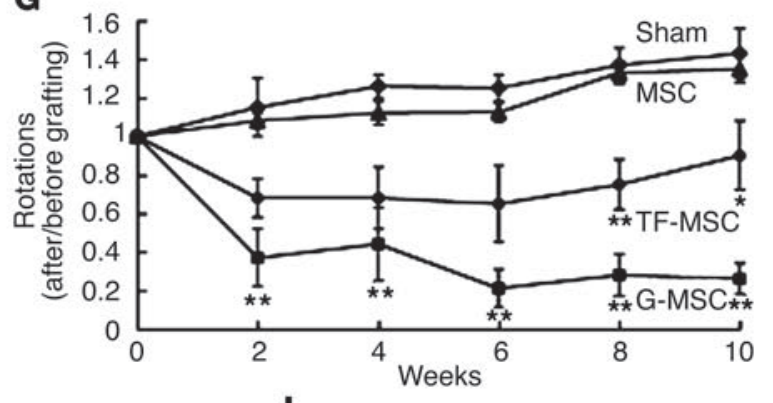

H

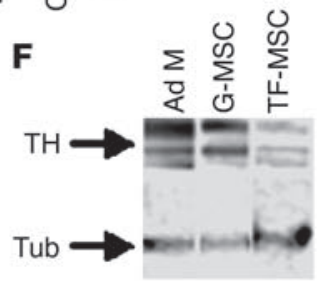

J

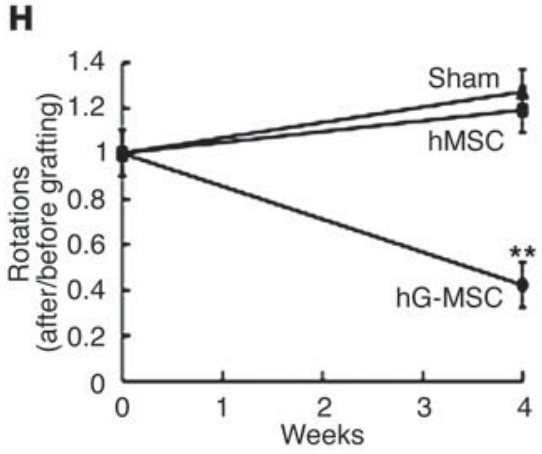

I
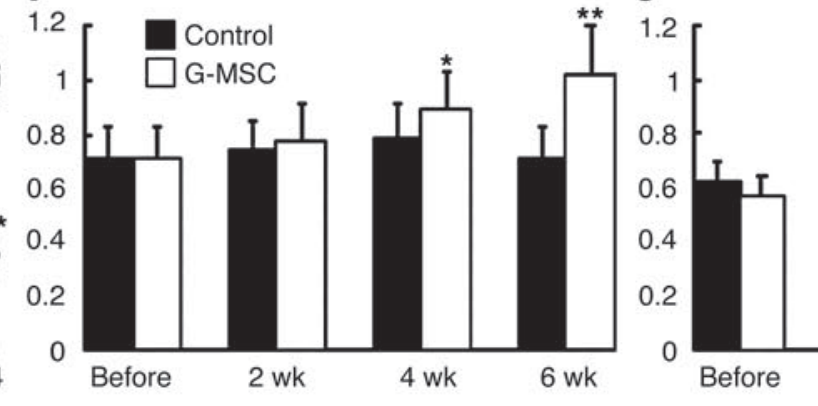

\section{Figure 5}

Induction of TH-positive cells. (A) The percentage of each transmitter in rat TF-MSCs and TH in rat G-MSCs. NPY, neuropeptide Y; CGRP, calcitonin gene-related peptide; VACht, vesicular acetylcholine transporter; SP, substance P; Glu, glutamate; Ser, serotonin transporter; Vip, vasoactive intestinal peptide; GABA, $\gamma$-aminobutyric acid. (B-D) TH expression (green) in rat (B) and human G-MSCs (C and D). Bars in B and $\mathbf{C}=100 \mu \mathrm{m} ; \mathbf{D}=30 \mu \mathrm{m}$. (E) RT-PCR of each factor in rat cells. Upregulation is recognized in G-MSCs compared with TF-MSCs. (F) Western blot of TH. Adrenal medulla (Ad M), as a positive control. $\beta$-tubulin (tub) as a loading control. (G) Analysis of apomorphine-induced rotations after transplantation of rat cells. Sham operation group (diamonds), MSC group (triangles), TF-MSC group (circles), and G-MSC group (squares) $\left({ }^{*} 0.01<P<0.05 ;{ }^{* *} P<0.01\right.$, statistical difference to sham group). (H) Apomorphine-induced rotation in sham-operated rats (triangles) and after the transplantation of human MSCs (hMSCs; squares) and human G-MSCs (hG-MSCs; circles). (I and J) Adjusting step test (I) and paw-reaching test (J) after transplantation of rat G-MSCs. Adjusting step test: the ratios of the number of steps of the lesioned side to the contralateral intact side paw. Paw-reaching test: the ratios of the number of pellets eaten by the lesioned side paw to the contralateral intact side paw. Control group received no grafting after 6-OHDA administration.

shown). Induced cells did not show neuronal features, and the ratio of MAP-2ab-positive cells was extremely low (8.5\% $\pm 0.6 \%$ ) (Figure $4, \mathrm{E}$ and F), suggesting that STAT downregulation is closely related to the induction of MAP-2ab-positive cells from MSCs.

Hes 1 and Hes 5 are known to be transcribed in response to Notch signaling and mediate many of the primary effects of Notch activation $(18,19)$. We transfected rat MSCs with Hes1 and Hes5 instead of NICD, followed by trophic factor induction, to examine whether Hes, downstream of Notch, is directly involved in the induction of MAP-2ab-positive cells. This failed to induce a neuronlike morphology (Figure 4, G and H) and MAP-2ab-positive cells were hardly detected (not shown). Accordingly, the ability of NICD to drive MSCs to MAP-2ab cells after trophic factor induction seems to be independent of Hes 1 and Hes5 activity.
Generation of TH-positive cells and transplantation to model rats of Parkinson's disease. We performed immunocytochemical examination of transmitters and related proteins in TF-MSCs (Figure 5A). GDNF is known to be involved with the generation and development of midbrain dopaminergic neurons (20). We considered whether administration of GDNF to TF-MSCs could induce TH-positive cells. We refer to TF-MSCs administered GDNF as "G-MSCs." We found that the proportion of cells immunopositive for TH rose from $3.9 \% \pm 0.6 \%$ in TF-MSCs to $41.0 \% \pm 14.1 \%$ in G-MSCs (Figure 5, A and B). TH-positive cells were also observed in human G-MSCs (Figure 5, C and D). GDNF treatment also elevated expression of Nurr-1, Lmx1b, En1, Ptx3, which are the transcription factors that have roles in the differentiation of midbrain precursors into dopaminergic neurons (21) (Figure 5E). 
Table 2

The number of rotations in each animal in sham, MSC, TF-MSC, and G-MSC groups before and 10 weeks after transplantation

\begin{tabular}{|c|c|c|c|c|c|c|c|c|c|c|c|}
\hline \multicolumn{3}{|c|}{ Sham group } & \multicolumn{3}{|c|}{ MSC group } & \multicolumn{3}{|c|}{ TF-MSC group } & \multicolumn{3}{|c|}{ G-MSC group } \\
\hline & Before & 10 weeks & & Before & 10 weeks & & Before & 10 weeks & & Before & 10 weeks \\
\hline No. 1 & 10 & 19 & No. 1 & 8 & 10 & No. 1 & 11 & 11 & No. 1 & 11 & 5 \\
\hline No. 2 & 9 & 15 & No. 2 & 10 & 13 & No. 2 & 7 & 3 & No. 2 & 12 & 0 \\
\hline No. 3 & 10 & 9 & No. 3 & 7.5 & 12 & No. 3 & 12.5 & 10 & No. 3 & 10 & 1 \\
\hline No. 4 & 13 & 18 & No. 4 & 10 & 11.5 & No. 4 & 11 & 16 & No. 4 & 10 & 2 \\
\hline No. 5 & 11 & 17 & No. 5 & 8 & 12 & No. 5 & 7 & 4 & No. 5 & 9.5 & 1 \\
\hline No. 6 & 14 & 18 & & & & No. 6 & 10 & 9 & No. 6 & 7 & 0 \\
\hline & & & & & & & & & No. 7 & 13 & 2.5 \\
\hline & & & & & & & & & No. 8 & 12.5 & 5 \\
\hline & & & & & & & & & No. 9 & 14 & 10 \\
\hline & & & & & & & & & No. 10 & 12 & 5 \\
\hline
\end{tabular}

Western blot analysis further confirmed these results (Figure $5 F)$. The amount of dopamine released upon depolarization was measured by HPLC using a reverse-phase column and an electrochemical detector system. G-MSC cells released $1.1 \mathrm{pmol} / 10^{6}$ cells of dopamine to the culture media in response to high $\mathrm{K}^{+}$depolarizing stimuli, whereas dopamine release from MSCs was under the detection level (data not shown).

To explore the ability of TF-MSCs and G-MSCs to survive and function in vivo, both rat and human cells were separately transplanted into the striatum of rats with a model of Parkinson disease. Unilateral administration of 6-hydroxy dopamine (6-OHDA) into the left medial forebrain bundle selectively destroys dopaminergic neurons in the substantia nigra, leading to quantifiable rotational behavior and providing a useful and commonly used model of Parkinson disease. Three types of rat cells labeled with retrovirus GFP (22) were transplanted: (a) MSCs (MSC group), (b) TF-MSCs (TF-MSC group), (c) G-MSCs (G-MSC group). Apomorphineinduced rotational behavior (mean rotation index) was examined every 2 weeks until 10 weeks after cell implantation. Sham-operated and MSC groups showed a rotational bias away from the lesioned side, which persisted, whereas the TF-MSC group showed slight improvement over time. In contrast, the G-MSC group demonstrated substantial recovery from rotation behavior (Figure 5G). The absolute number of rotations in each animal in each group is shown in Table 2. Furthermore, nonpharmacological behavior tests, stepping behavior and paw reaching, were assessed and quantified. Four and 6 weeks after grafting of G-MSCs, these rats showed significant improvement in step adjustment (Figure 5I) and paw reaching (Figure 5J). Three transplanted animals from the G-MSC group were followed for up to 16 weeks, and there was no tumor formation observed in the brain.

\section{Figure 6}

Histological analysis of striatum 10 weeks after transplantation in GMSC group. (A) GFP-labeled rat transplanted cells were found in the gray regions in the schematic diagrams. (B) Box $\mathbf{B}$ in panel $\mathbf{A}$ is subjected to HE staining. Implantation zone is indicated by a dotted line. Bar $=100 \mu \mathrm{m}$. (C) Immunostaining of anti-GFP (green) and -TH (red) of box $\mathbf{C}$ in panel $\mathbf{B}$ from an adjacent section of panel $\mathbf{B}$. TH-positive processes are extending to the outside of the implantation zone (arrows). Bar = $30 \mu \mathrm{m}$. (D-G) Immunostaining for TH (D), DAT (E), GFAP $(\mathbf{F})$, and $\mathrm{O} 4(\mathbf{G})$ (all red color-coded) taken from the area of boxes $\mathbf{D}-\mathbf{G}$ in panel $\mathbf{A}$. Bar $=50 \mu \mathrm{m}$.
Ten weeks following grafting to the Parkinson disease model brains (G-MSC group), immunohistochemistry was carried out. In serial sections of the G-MSC group, grafted GFP-labeled cells were found to migrate and extend beyond the injected site (Figure 6A). Approximately $3.4 \times 10^{4}(34 \%)$ cells were counted in the striatum. $\mathrm{TH}$-positive processes extended to the outside of the implantation zone (Figure 6, B and C). The grafted striatum showed migration of GFP-positive transplanted cells with positivity for neurofilaments (not shown), TH, and dopamine transporter (DAT) (Figure $6, \mathrm{D}$ and $\mathrm{E}$ ). Immunoreactivities among GFP-labeled cells to TH and DAT were $45.7 \% \pm 4.2 \%$ and $30.7 \% \pm 0.9 \%$, respectively. Most of GFP-labeled cells were negative to GFAP (Figure 6F), although $2.5 \% \pm 1.4 \%$ of cells were recognized to be GFAP positive. O4-positive cells could not be recognized (Figure 6G).

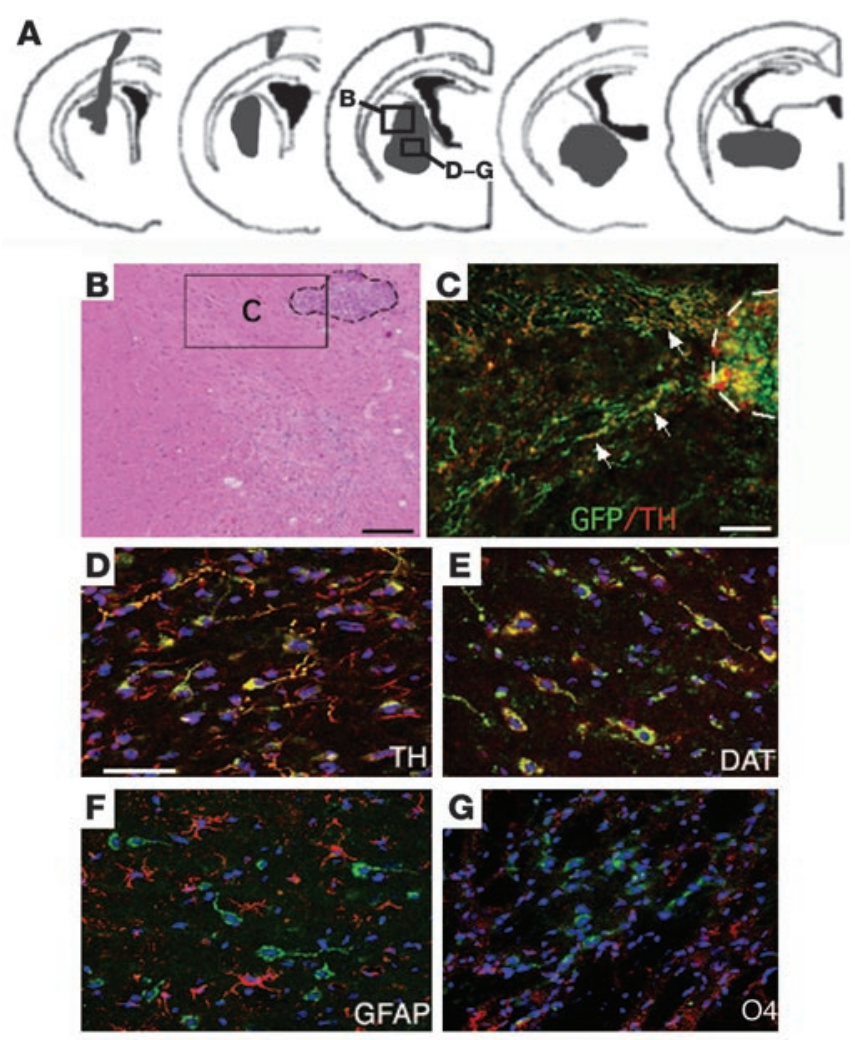


Human G-MSCs were similarly transplanted into the striatum of 6-OHDA-lesioned rats. Animals were immunosuppressed with FK 506 daily, and rotational behavior was recorded at 4 weeks. Grafting resulted in significant improvement in rotational behavior (Figure $5 \mathrm{H}$ ). Rotation indexes of six individual rats 4 weeks after sham operation were $1.4,1.4,1.3,1.2,1.1$, and 1.2 (mean rotation $1.26 \pm 0.1$ ), and indexes for four individual rats after transplantation of human MSCs were $0.9,1.3,1.1$, and 1.4 , respectively (mean rotation $1.20 \pm 0.2$ ). Results from five individual rats after grafting of human G-MSCs were $0.75,0.3,0.3,0.5$, and 0.28 (mean rotation $0.43 \pm 0.2, P<0.01$ ). The capacity of grafted human G-MSCs to synthesize and release dopamine was assessed by measuring dopamine concentration in the culture medium of slices of transplanted brain by HPLC. Brain slices were divided half at the midline into engrafted and intact untransplanted sides and cultured separately. The dopamine concentration in each culture medium was measured, and the ratio of dopamine concentration (lesioned or engrafted side/intact side) was calculated. This ratio was $0.57 \pm 0.01$ in control rats (who did not receive cell grafting after 6-OHDA lesion) $(n=4)$, compared with $0.67 \pm 0.04$ in engrafted animals $(n=4)$, a significant $(P=0.04)$ increase in dopamine with transplantation.

\section{Discussion}

In this study, we showed that NICD-transfection followed by trophic factor induction efficiently induced MSCs with neuronal characteristics in an ordered and large-scale fashion. Further treatment of these induced cells with GDNF substantially increased the proportion of TH-positive and dopamineproducing cells, and functional improvement could be achieved when grafted in a rodent model of Parkinson disease. A major glial marker, GFAP, was not detected in our induced cells by immunocytochemistry, Western blotting, and RT-PCR, and its promoter activity was extremely low, confirming that the existence of glial cells among the induced cell population is extremely unlikely. In contrast to previous studies using ES cells, NSCs/ NPCs, and MSCs, our induction system is virtually devoid of glial elements and therefore would have a great clinical advantage. Furthermore, induced postmitotic functional neurons might be better suited for clinical application since they, at least theoretically, may minimize the risk of tumor formation.

Our experiments have also shown that N-MSCs expressed GLAST and 3-PGDH, which are known to be expressed in NSC/ NPC. These N-MSCs could differentiate into cells with neuronal characteristics after application of trophic factors such as bFGF, CNTF, and FSK. It has been suggested that bFGF and FSK activate the MAPK cascade, which in turn activates transcription of neuronal genes $(23,24)$. Previous reports have suggested that gp130, which mediates signals from CNTF, leukemia inhibitory factor, and IL-6, activates both JAK-STAT- and MAPK-signaling pathways (25). The present experiments showed that CNTF had an action on N-MSCs in which STAT1 and STAT3 were not expressed, suggesting that CNTF may have acted via MAPK pathway and had a synergistic effect toward neuronal induction when combined with stimuli of bFGF and FSK.

JAK/STAT inhibitor administration and constitutive active STAT $1 / 3$ transfection showed that downregulation of STATs was tightly associated with NICD-mediated neuronal induction, whereas Hes, downstream of Notch, was not involved in the induction event. Whether these NICD-mediated responses reflect some physiological aspects of MSCs or not remains to be clarified. Since the neuronlike cells could be induced, to an extent, with JAK/STAT inhibitor instead of the NICD transfection, it might be possible to efficiently generate neurons without gene transfection. This may have advantages for possible future clinical application.

MSCs are originally known to produce many kinds of trophic factors (26). We performed Western blot analysis to investigate whether G-MSCs and MSCs were producing trophic factors such as BDNF and bFGF. The experiment showed that both types of cells produced BDNF and bFGF to the similar extent (data not shown). There exists a possibility that trophic factors released from transplanted cells may contribute to the functional recovery of the Parkinson model rats. Behavioral recovery, however, may not be due simply to trophic support, because dopamine release was recognized in G-MSCs while it was under detection level in MSCs, and the G-MSC group showed statistically significant recovery of rotational behavior compared with MSC transplantation (namely, the MSC group).

Cell fusion has recently been proposed as an explanation for stem cell plasticity, including marrow stromal cells, raising questions about its mechanisms of transdifferentiation in vivo $(27,28)$. Our studies showed that MSCs were manipulated in the culture system to differentiate into postmitotic cells with neuronal characteristics and behave as such in vivo, so the present results may not simply be attributed to stem cell fusion.

MSCs have a great potential as therapeutic agents against neurological diseases since they can be readily obtained through a wellestablished clinical procedure and are easy to isolate and expand for autotransplantation with no risk of rejection. In this study, we have succeeded in obtaining the high ratio of neuronal cells. Further studies are needed, however, to ensure the long-term safety and efficacy of manipulated MSCs.

\section{Methods}

Culture of MSCs. All animal experiments were approved by the Animal Care and Experimentation Committee of Kyoto University Graduate School of Medicine and Yokohama City University School of Medicine.

We used MSCs from rat and human sources in this study. Rat (Wistar strain) MSCs were isolated and cultured as described in our previous report (22). Human MSCs from two different sources were used separately (never mixed) for each experiment: one from a commercially available source (PT-2501; BioWhittaker Inc., Walkersville, Maryland, USA) and the other from a single healthy donor (informed consent obtained according to Guidelines of the Ethics Committee of the Kyoto University Graduate School and Faculty of Medicine). For the clonal expansion of rat MSCs, cells were subcloned by limiting dilution as described (9).

FACS analysis. MSCs were incubated with FITC-labeled antiCD34 (Santa Cruz Biotechnology Inc., Santa Cruz, California, USA), -CD54, -CD90, -CD11b/c, -CD29, or -vWF (PharMingen, San Diego, California, USA). Data were acquired and analyzed on FACScalibur with CellQuest software (Becton Dickinson and Co., Franklin Lakes, New Jersey, USA).

Plasmids. The NICD cDNA used in this experiment coded for a transmembrane region that included a small fragment of extracellular domain, followed by a sequence encoding the entire intracellular domain of mouse Notch (initiating at amino acid 1703 and terminating at the $3^{\prime}$ untranslated sequence) (29). This fragment was subcloned into a pCI-neo vector (Promega Corp., 
Madison, Wisconsin, USA) and was transfected with MSCs using Lipofectamine 2000 and selected by G418 for 11 days, according to manufacturer's instruction (Invitrogen Corp., Carlsbad, California, USA). Also transfected with MSCs using Lipofectamine 2000 were pNICD-IRES2-EGFP, mouse Hes1 and Hes5 (19) subcloned into pCI-neo, pEF-BOS FLAG-mouse STAT3 (14), luciferase reporter plasmids of 3-PGDH (both full-length and M1965) (10), NeuroD (15), and GFAP promoter (16). For reporter assays, the dual luciferase assay kit (Promega Corp.) was used.

Trophic factor induction. For trophic factor induction, 60-70\% confluent cultures of NICD-transfected MSCs were incubated in $\alpha$-MEM containing 10\% FBS, $5 \mu$ M FSK (Calbiochem, La Jolla, California, USA), $10 \mathrm{ng} / \mathrm{ml}$ bFGF (Peprotech Ec Ltd., London, United Kingdom), and $10 \mathrm{ng} / \mathrm{ml} \mathrm{CNTF} \mathrm{(R \& D} \mathrm{Systems} \mathrm{Inc.,} \mathrm{Min-}$ neapolis, Minnesota, USA). For GDNF treatment, $50 \mathrm{ng} / \mathrm{ml}$ of GDNF (Peprotech Ec Ltd.) was administered into $\alpha$-MEM containing $10 \%$ FBS culture medium. As for the differentiation of induced neuronal cells in the electrophysiological analysis, medium was replaced with $\alpha$-MEM containing $10 \%$ FBS, $50 \mathrm{ng} / \mathrm{ml}$ BDNF (Peprotech Inc., Rocky Hill, New Jersey, USA), and $50 \mathrm{ng} /$ $\mathrm{ml}$ of NGF (Takara Shuzo Co., Tokyo, Japan) 7 days after trophic factor induction. For the JAK inhibitor experiment, $40 \mu \mathrm{g} / \mathrm{ml}$ of 4-(4'-hydroxyphenyl)amino-6,7-dimethoxyquinazoline (WHI-P131; Calbiochem) was added to a culture medium of $\alpha$-MEM containing $10 \%$ FBS for 2 days.

RT-PCR analysis. To analyze relative expression of different mRNAs, the amount of cDNA was normalized based on the signal from ubiquitously expressed $\beta$-actin. Primer sequences and precise conditions are described elsewhere $(1,17,19,30)$. Positive control for each factor is as follows; Notch extracellular domain, NICD, Mash1, Hes1, Hes5, STAT1, STAT3, GFAP, and $\beta$-actin are from total RNA of rat brain, Math1 is from cerebellum, and Neurogenin 1 is from spinal cord.

$A b$ 's. The following primary Ab's were used: CD31, CD45, PDGF-receptor $\beta$, nestin (PharMingen), glutamate transporter GLAST (11), 3-PGDH (10), MAP-2ab, GFP, smooth muscle actin, $\beta$-tubulin isotype 3 , FLAG, pan sodium channel, $\gamma$-aminobutyric acid, $\beta$-tubulin (for Western blot analysis) (Sigma-Aldrich, St. Louis, Missouri, USA), rabbit antineurofilament-M, TH, GalC, serotonin transporter, vesicular acetylcholine transporter, glutamate, DAT (Chemicon International, Temecula, California, USA), GFAP (DAKO Corp., Carpinteria, California, USA), O4 (Boehringer Mannheim $\mathrm{GmbH}$, Mannheim, Germany), neuropeptide Y (Peninsula Laboratories Inc., Belmont, California, USA), vasoactive intestinal peptide (Incstar Corp., Stillwater, Minnesota, USA), collagen type II (LSL, Tokyo, Japan), calcitonin gene-related peptide, substance $\mathrm{P}$ (Amersham International, Amersham, United Kingdom), goat antineurofilament-M (for triple staining with MAP-2ab and $\beta$-tubulin isotype 3), Ki67 (Serotec Ltd., Kidlington, United Kingdom), Notch extracellular domain, and NICD (Santa Cruz Biotechnology Inc.). Precise procedures for immunocytochemistry (22) and Western blot analysis (31) were described previously. Inspections for immunocytochemistry and immunohistochemistry were made under a confocal laser scanning microscope (Radians 2000; Bio-Rad Laboratories Inc., Hemel Hempstead, United Kingdom). As for the Western blot, all lanes presented are from the same Western blot analysis. The degree of positive cells was expressed through the ratios of immunopositive cells in relation to the total number of nuclei stained with TOTO-3 iodide (Molecular Probes Inc., Eugene, Oregon, USA).
As for Brd-U labeling, Brd-U $(10 \mu \mathrm{M})$ was added to the culture medium for 24 hours after trophic factor induction (5 days) and stained with anti-Brd-U (Boehringer Mannheim $\mathrm{GmbH}$ ).

Electrophysiological recording. In the whole-cell patch clamp, cells with a relatively large cell body and neuritelike structures were chosen for recording. Delayed rectifier potassium currents were measured as described previously (31). Currents were elicited by step depolarization of the membrane in $10-\mathrm{mV}$ increments for 50 milliseconds from -50 to $+50 \mathrm{mV}$ from the holding potential of $-100 \mathrm{mV}$. Fast sodium currents were measured in artificial cerebrospinal fluid (ACSF) as described previously (32). The composition of ACSF is as follows (in $\mathrm{mM}$ ): $125 \mathrm{NaCl}, 2.5 \mathrm{KCl}$, $2 \mathrm{CaCl}_{2}, 1 \mathrm{MgCl}_{2}, 26 \mathrm{NaHCO}_{3}, 1.25 \mathrm{NaH}_{2} \mathrm{PO}_{4}$, and 17 D-glucose saturated by $95 \% \mathrm{O}_{2} / 5 \% \mathrm{CO}_{2}$. For the Na current/TTX $(1 \mu \mathrm{M})$ experiment, a patch pipette was filled with Cs-based internal medium as follows (in mM): 120 Cs-gluconate, 10 HEPES, 0.5 EGTA, $20 \mathrm{CsCl}$ (adjusted to pH 7.2). Na current was generated every 25 seconds by 20 -millisecond voltage steps to $-35 \mathrm{mV}$ from the holding potential of $-80 \mathrm{mV}$ (liquid junction potential of $-10 \mathrm{mV}$ was corrected) recorded at room temperature $\left(23^{\circ} \mathrm{C}\right)$. Action potential was recorded with $\mathrm{K}$-based internal medium in ACSF at $33^{\circ} \mathrm{C}$. The composition of K-based internal medium is as follows (in mM): $120 \mathrm{~K}$-gluconate, 10 HEPES, 0.5 EGTA, 20 $\mathrm{KCl}$ (adjusted to $\mathrm{pH} 7.2$ ).

Dopamine release assay of cultured cells. The experiment was performed as described previously (33). Cells were washed twice with a low $\mathrm{K}^{+}$solution (in mM: $20 \mathrm{HEPES}, 140 \mathrm{NaCl}, 4.7 \mathrm{KCl}, 2.5 \mathrm{CaCl}_{2}$, $1.2 \mathrm{MgSO}_{4}, 1.2 \mathrm{KH}_{2} \mathrm{PO}_{4}$, and 11 glucose, $\mathrm{pH} 7.4$ ), incubated in the low $\mathrm{K}^{+}$solution for 2 minutes, and then the medium was replaced with high $\mathrm{K}^{+}$solution (same as low $\mathrm{K}^{+}$solution except for $85 \mathrm{NaCl}$ and $60 \mathrm{KCl}$ ) for 5 minutes. Concentration of dopamine was determined by HPLC using a reverse-phase column and an electrochemical detector system (Eicom, Kyoto, Japan). The mobile phase was composed of $0.1 \mathrm{M}$ phosphate buffer ( $\mathrm{pH} 6.0$ ), 20\% methanol, 500 $\mathrm{mg} / \mathrm{l}$ octanesulfonic acid, $50 \mathrm{mg} / \mathrm{l}$ disodium EDTA. The injection volume was $20 \mu \mathrm{l}$, and the working electrode was set at $0.45 \mathrm{~V}$ compared with an $\mathrm{Ag} / \mathrm{AgCl}$ reference electrode (33).

Analysis of Parkinson disease model rats. The procedures for creating the model were described in our previous report (34). In brief, adult male Wistar rats (weighing 250-300 g) were anesthetized with sodium pentobarbital (40 mg/ $\mathrm{kg}$, intraperitoneally) and 6-OHDA ( $8 \mu \mathrm{g} / 4 \mu \mathrm{l}$ of $0.1 \%$ ascorbate-saline) was injected into the left medial forebrain bundle (anteroposterior $[\mathrm{A} / \mathrm{P}]=-4.4 \mathrm{~mm}$; lateral $[\mathrm{L}]=+1.1 \mathrm{~mm}$ from bregma, ventral $[\mathrm{V}]=-7.7 \mathrm{~mm}$ from dura). Rats showing an average of fewer than six turns per minute for the first 30 minutes after apomorphine administration $(0.8 \mathrm{mg} / \mathrm{kg}$, subcutaneously) were excluded from the transplantation experiments. Cells $\left(10^{5}\right.$ cells $\left./ 8 \mu \mathrm{l}\right)$ were grafted into the ipsilateral striatum (left side) following coordinates: $\mathrm{A} / \mathrm{P}=+0.5$ $\mathrm{mm} ; \mathrm{L}=+3.0 \mathrm{~mm}$ from bregma, and $\mathrm{V}=-4.5 \mathrm{~mm}$. The infusion syringe was placed at the same coordinates unilaterally for shamoperation rats, but no fluid was infused. The number of animals was as follows: sham-operation, six; MSC group, five; TF-MSC group, six; G-MSC group, ten. The mean rotation number was calculated at each time point, and then mean rotation index (the mean rotation number for after grafting and before grafting) was obtained. Adjusting step test (35) and paw-reaching test (36) were performed as described previously, and the control group received no grafting after 6-OHDA administration. Four animals were used for each group. 
In the case of human MSC transplantation, five animals were grafted and immunosuppressed by subcutaneous injection of FK506 (1 mg/kg; Fujisawa Pharmaceutical Co. Ltd., Osaka, Japan) every day. For dopamine measurement in brain slices, 1-mm-thick coronal brain slices were obtained (A/P $+2.5 \mathrm{~mm}$ to $-1.5 \mathrm{~mm}$ from bregma; four slices total), separated at the midline, and each side cultured separately in $\alpha$-MEM containing $10 \%$ FBS. Twenty-four hours later, culture media of brain slices were collected and HPLC analysis performed by SRL Communication and Health, Tokyo, Japan.

Statistical analysis. Data are expressed as mean plus or minus SEM. Data were compared using ANOVA with pairwise comparisons using the Bonferroni method.

\section{Acknowledgments}

We are grateful to H. Ohmori (Department of Physiology and Neurobiology, Kyoto University) for collecting electrophysiological data, M. Kawaichi (Nara Institute of Science and Technology), S. Furuya (Brain Science Institute), C.E. Finch (University of Southern California, Los Angeles), M. Watanabe (Hokkaido University),
M.J. Tsai (Baylor College of Medicine), R. Kageyama (Kyoto University), T. Taga (Kumamoto University) for providing cDNAs and Ab's, and to Issei Nagaki (Yokohama City University) and Masami Yoshida (Kyoto University) for technical assistance. We also express our cordial thanks to Krys Bankiewicz (University of California, San Francisco) and Laszlo Tamas (Pacific Neurosciences Institute) for their valuable advice and review of the manuscript. This work was supported by a Grant-in-Aid for Scientific Research on Priority Areas-Advanced Brain Science Project (14017079, 15016091) and $(15590166,14370008)$ from the Ministry of Education, Culture, Sports, Science and Technology, Japan.

Received for publication December 29, 2003, and accepted in revised form April 20, 2004.

Address correspondence to: Mari Dezawa, Department of Anatomy and Neurobiology, Kyoto University Graduate School of Medicine, Yoshida Konoe-cho, Sakyo-ku, Kyoto 606-8501, Japan. Phone: 81-75-753-4343; Fax: 81-75-751-7286; E-mail: dezawa@anat2.med.kyoto-u.ac.jp.
1. Kawasaki, H., et al. 2000. Induction of midbrain dopaminergic neurons from ES cells by stromal cell-derived inducing activity. Neuron. 28:31-40.

2. Pittenger, M.F., et al. 1999. Multilineage potential of adult human mesenchymal stem cells. Science. 284:143-147.

3. Woodbury, D., Schwarz, E.J., Prockop, D.J., and Black, I.B. 2000. Adult rat and human bone marrow stromal cells differentiate into neurons. J. Neurosci. Res. 61:364-370.

4. Sanchez-Ramos, J.R. 2002. Neural cells derived from adult bone marrow and umbilical cord blood. J. Neurosci. Res. 69:880-893.

5. Jiang, Y., et al. 2002. Pluripotency of mesenchymal stem cells derived from adult marrow. Nature. 418:41-49.

6. Lundkvist, J., and Lendahl, U. 2001. Notch and the birth of glial cells [review]. Trends Neurosci. 24:492-494.

7. Gaiano, N., Nye, J.S., and Fishell, G. 2000. Radial glial identity is promoted by Notch 1 signaling in the murine forebrain. Neuron. 26:395-404.

8. Morrison, S.J., et al. 2000. Transient Notch activation initiates an irreversible switch from neurogenesis to gliogenesis by neural crest stem cells. Cell. 101:499-510.

9. Kohyama, J., et al. 2001. Brain from bone: efficient "meta-differentiation" of marrow stroma-derived mature osteoblasts to neurons with Noggin or a demethylating agent. Differentiation. 68:235-244.

10. Yamasaki, M., et al. 2001. 3-Phosphoglycerate dehydrogenase, a key enzyme for L-serine biosynthesis, is preferentially expressed in the radial glia/astrocyte lineage and olfactory ensheathing glia in the mouse brain. J. Neurosci. 21:7691-7704.

11. Shibata, T., et al. 1997. Glutamate transporter GLAST is expressed in the radial glia-astrocyte lineage of developing mouse spinal cord. J. Neurosci. 17:9212-9219.

12. Grosse, G., et al. 2000. Expression of Kv1 potassium channels in mouse hippocampal primary cultures: development and activity-dependent regulation. J. Neurosci. 20:1869-1882.

13. Vicario-Abejon, C., Collin, C., McKay, R.D., and Segal, M. 1998. Neurotrophins induce formation of functional excitatory and inhibitory synapses between cultured hippocampal neurons. J. Neurosci.
18:7256-7271.

14. Nakashima, K., et al. 2001. BMP2-mediated alteration in the developmental pathway of fetal mouse brain cells from neurogenesis to astrocytogenesis. Proc. Natl. Acad. Sci. U. S. A. 98:5868-5873.

15. Peyton, M., et al. 1996. BETA3, a novel helix-loophelix protein, can act as a negative regulator of BETA2 and MyoD-responsive genes. Mol. Cell. Biol. 16:626-633.

16. Rozovsky, I., et al. 2002. Estradiol (E2) enhances neurite outgrowth by repressing glial fibrillary acidic protein expression and reorganizing laminin. Endocrinology. 143:636-646.

17. Seta, Y., Toyono, T., Takeda, S., and Toyoshima, K 1999. Expression of Mash1 in basal cells of rat circumvallate taste buds is dependent upon gustatory innervation. FEBS Lett. 444:43-46.

18. Ishibashi, M., et al. 1994. Persistent expression of helix-loop-helix factor HES-1 prevents mammalian neural differentiation in the central nervous system. EMBO J. 13:1799-1805.

19. Zheng, J.L., Shou, J., Guillemot, F., Kageyama, R., and Gao, W.Q. 2000. Hes1 is a negative regulator of inner ear hair cell differentiation. Development. 127:4551-4560.

20. Akerud, P., Alberch, J., Eketjall, S., Wagner, J., and Arenasm, E. 1999. Differential effects of glial cell line-derived neurotrophic factor and neurturin on developing and adult substantia nigra dopaminergic neurons. J. Neurochem. 73:70-78.

21. Sakurada, K., Ohshima-Sakurada, M., Palmer, T.D., and Gage, F.H. 1999. Nurr1, an orphan nuclear receptor, is a transcriptional activator of endogenous tyrosine hydroxylase in neural progenitor cells derived from the adult brain. Development. 126:4017-4026.

22. Dezawa, M., Takahashi, I., Esaki, M., Takano, M., and Sawada, H. 2001. Sciatic nerve regeneration in rats induced by transplantation of in vitro differentiated bone-marrow stromal cells. Eur. J. Neurosci. 14:1771-1776.

23. Neufeld, B., et al. 2000. Serine/threonine kinases $3 \mathrm{pK}$ and MAPK-activated protein kinase 2 interact with the basic helix-loop-helix transcription factor E47 and repress its transcriptional activity. J. Biol. Chem. 275:20239-20242.

24. Stork, P.J., and Schmitt, J.M. 2002. Crosstalk between
cAMP and MAP kinase signaling in the regulation of cell proliferation. Trends Cell Biol. 12:258-266.

25. Ihara, S., et al. 1997. Dual control of neurite outgrowth by STAT3 and MAP kinase in PC12 cells stimulated with interleukin-6. EMBOJ. 16:5345-5352.

26. Chen, X., et al. 2002. Human bone marrow stromal cell cultures conditioned by traumatic brain tissue extracts: growth factor production. J. Neurosci. Res. 69:687-691.

27. Terada, N., et al. 2002. Bone marrow cells adopt the phenotype of other cells by spontaneous cell fusion. Nature. 416:542-545.

28. Alvarez-Dolado, M., et al. 2003. Fusion of bonemarrow-derived cells with Purkinje neurons, cardiomyocytes and hepatocytes. Nature. 425:968-973.

29. Yamamoto, N., et al. 2001. Role of Deltex-1 as a transcriptional regulator downstream of the Notch receptor. J. Biol. Chem. 276:45031-45040.

30. Schwaiger, F.W., et al. 2000. Peripheral but not central axotomy induces changes in Janus kinases (JAK) and signal transducers and activators of transcription (STAT). Eur. J. Neurosci. 12:1165-1176.

31. Murata, H., et al. 2002. Von Hippel-Lindau tumor suppressor protein transforms human neuroblastoma cells into functional neuron-like cells. Cancer Res. 62:7004-7011.

32. Toda, H., Takahashi, J., Mizoguchi, A., Koyano, K., and Hashimoto, N. 2000. Neurons generated from adult rat hippocampal stem cells form functional glutamatergic and GABAergic synapses in vitro. Exp. Neurol. 165:66-76.

33. Nagano, Y., et al. 2003. Siah-1 facilitates ubiquitination and degradation of synphilin-1. J. Biol. Chem. 278:51504-51514.

34. Yamada, H., et al. 2003. Transfer of the von Hippel-Lindau gene to neuronal progenitor cells in treatment for Parkinson's disease. Ann. Neurol. 54:352-359.

35. Olsson, M., Nikkhah, G., Bentlage, C., and Bjorklund, A. 1995. Forelimb akinesia in the rat Parkinson model: differential effects of dopamine agonists and nigral transplants as assessed by a new stepping test. J. Neurosci. 15:3863-3875.

36. Montoya, C.P., Campbell-Hope, L.J., Pemberton, K.D., and Dunnett, S.B. 1991. The "staircase test": a measure of independent forelimb reaching and grasping abilities in rats. J. Neurosci. Methods. 36:219-228. 